\title{
Mobile-Based Medical Emergency Ambulance Scheduling System
}

\author{
Bassey Isong and Nosipho Dladlu \\ North-West University, Computer Science Department, Mafikeng, South Africa \\ E-mail: \{bassey.isong, nosipho.dladlu\}@nwu.ac.za \\ Tsholofelo Magogodi \\ North-West University, Computer Science Department, Mafikeng, South Africa \\ E-mail: tmag.tshoks@yahoo.com
}

\begin{abstract}
Effective and efficient public service delivery like healthcare services are very important in today's society, especially in the rural areas. People in rural areas are expected to have access to public facilities at all times. However, these services are not always available when they are needed. This paper discusses the problems faced by rural areas of Mafikeng in South Africa (SA) when public and basic healthcare facilities like medical ambulance transports are needed during emergency situation. The challenges ranges from poor communication, poor road network and unstructured address to non-arrival of ambulances leading loss of lives that are preventable. This paper designed and implemented a system prototype using mobile application technologies to offer cost-effective services to patients during emergencies. It is intended to reduce long queues in hospitals and long waiting periods for an ambulance via location-based services. By using this application, lives in the rural areas can be made easier and loss of lives prevented by providing timely response from the appropriate healthcare providers during emergencies.
\end{abstract}

Index Terms-Ambulance, Healthcare Service, Transport, Mobile, Rural Areas.

\section{INTRODUCTION}

The rapid advancements in information and communication technologies (ICTs) in today's e-society has become a significant element of development. Its profound impacts has been felt in every sector of many countries' economy such as education, communication, banking, healthcare, and so on. In particular, ICT tools has really changed the way information is created, stored, used and exchanged. An example is the availability and pervasiveness of powerful mobile devices like phones and so on that the world has witnessed for decades now. Previously, high technological devices were only owned by the elites or the wealthiest in the society but currently, mobile phones are available and thus accessible to the majority of the world's population [1]. In the perspective of healthcare, the integration and usage of ICTs has been valuable and commendable worldwide. However, in most rural areas, the impact is yet to be completely felt. In this case, the healthcare systems must operate to match its supply to the demands of those communities. Currently, several communities are experiencing inadequate match of healthcare supply impeded by poor transport systems and has severely compromise the optimal use of scarce human resources especially in South Africa (SA). Statistics has shown that about $46 \%$ of SAs' population resides in the rural communities, but they are served by only $12 \%$ of doctors and $19 \%$ of nurses [2]. Moreover, there are serious problems with the affordability, availability and reliability of transport in such rural areas [2]. Consequently, in the event of emergencies or nonemergencies, the available ambulances finds it difficult, if not impossible to access the needed patient's home [2]. The situation is even exacerbated when there are no structured addresses in place [2]. With this state of affairs, for a patient's referral, a delay of about two to three hours can be experienced before an ambulance arrives. Additionally, in the rural areas, establishing contact or having access to the ambulances is not an easy task since the call centers are only operational in some areas and always very busy. These has resulted to the loss of lives of many patients which are preventable if there were facilities in place to attend to their needs when needed. Providing a lasting solution to this present problem constitutes the motivation for this paper.

In this paper, we designed and implemented a system integrated with a mobile application and a location based service (LBS) [3][5] to bridge the communication gap between healthcare providers and patients residing in the rural areas. The focal point are the rural communities in Mafikeng in SA. With this system, patients in need of medical attention unavailable in the rural area of residence can perform tasks such as make an appointment or book for consultation in a hospital, access the services of ambulance transportation quickly, as well as predicting its arrival time. Furthermore, it will assist paramedics to locate the current or actual position of the patient and other healthcare providers like nurses and doctors who will attend to the patients in time. To this end, smart mobile devices are utilized for the efficient and effective operations. The objective of this paper is therefore, to improve the quality of healthcare services in the rural 
areas of Mafikeng where modern hospitals are not found. In particular, the research questions this paper seek to answer are: how can communication be improved between healthcare providers and patients? how can the time spent waiting for the ambulance and queues in hospital be reduced? We believe that having such systems where needed healthcare systems can't be found could go a long way to save the lives of millions of poor citizens that are in critical conditions.

The paper is organized as follows: Section II is the study background, Section III is the related works, Section IV is the proposed system architecture and Section V discusses the algorithm and prototype implementation. Section VI and VII are the paper discussions and conclusions respectively.

\section{BACKGROUND INFORMATION}

In recent years, the world has witnessed the explosive growth in ICTs which has become the ultimate drivers of development in today's e-society. Among such development is the impact that has been created in the provision of effective and efficient healthcare delivery. However, the impact has been one-sidedly felt since it is only enjoyed by people in the urban areas while majority of the rural areas are still in the "dark". This has resulted in the loss of thousands of lives that could have been prevented if needed facilities were available and accessible. In addition, the transport services that could have been a leverage, contributed negatively to the accessibility of such facilities. A particular situation that motivated for the solution presented in this paper is the healthcare challenges faced by people in the rural areas of Mafikeng in SA where basic and key healthcare facilities are absent. Moreover, poverty, lack of other basic public facilities such as police stations, libraries, and information centers such as public computers and Internet, very poor public transport services, lack of Emergency Medical Services (EMSs) and so on are the state of the affairs. Fig. 1 shows Mafikeng and its environs from Google Maps.

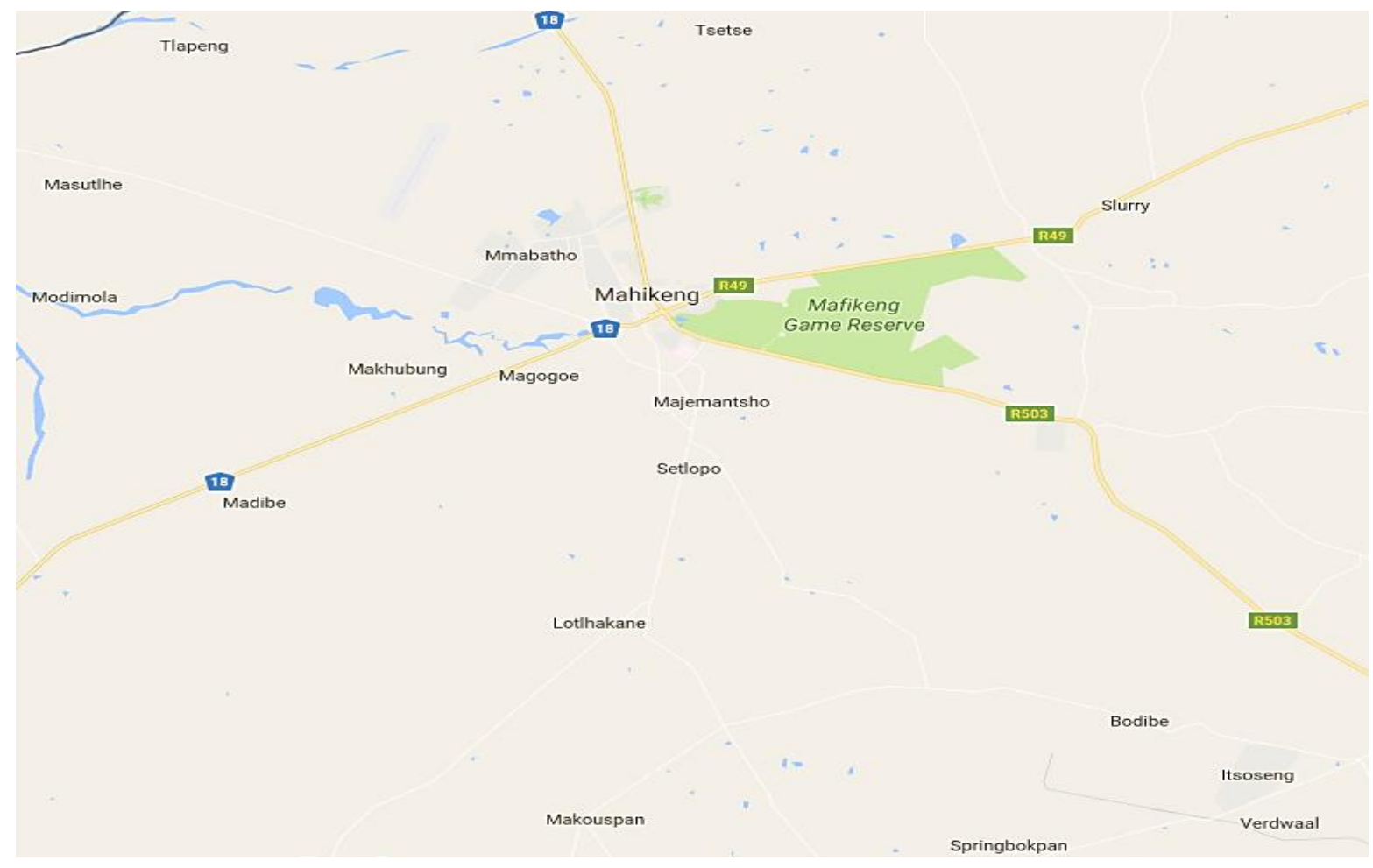

Fig.1. Mafikeng and its Environs from Google Maps

In particular, Mantsa village has experienced the worst challenge when it comes to accessing healthcare facilities especially during emergencies. In Mantsa village, we found that it was cumbersome for the inhabitants to have access to hospitals or health centers irrespective of the criticality of the problem. In most cases, the patients have to provide their own transports via private hire of which the process involved is complex, time consuming and could even results to deaths. Moreover, even if they could get to call ambulances, the supply is always outnumbered by the demand. For instance, for the need of about 70 ambulances, only 25 ambulances will be operational
[2][4]. Fig. 2 is an example of ambulance transport operating in SA. Moreover, coupled to the limited ambulance issues are the bad roads and inadequate transport in terms of regularity, reliability and cost as well as structured addresses. Bad roads has led to minimal investment in public transports as well as accessibility of ambulances in the rural areas like Mantsa. Consequently, people have to walk long distances waiting and looking for transport to take them to the hospital or other places of interest. The situation is even worst during bad weather when patients and or their caregivers will have to wait in pain or even die before they could reach 
the hospital. Also, majority of the residence are elderly people and children who are sometimes unaware of their exact location due to several reasons which may include a serious injury, shock or a criminal attack. In some cases, even if public transports or ambulances are contacted, they fail to reach their destination or the longest routes are always taken whilst there is available shorter alternatives. Even when ambulances struggled to find the specific dwelling [2][4], unstructured addresses also poses an impediment to the ambulance drivers since most of them are foreign to the rural environment and the communities are geographically dispersed.

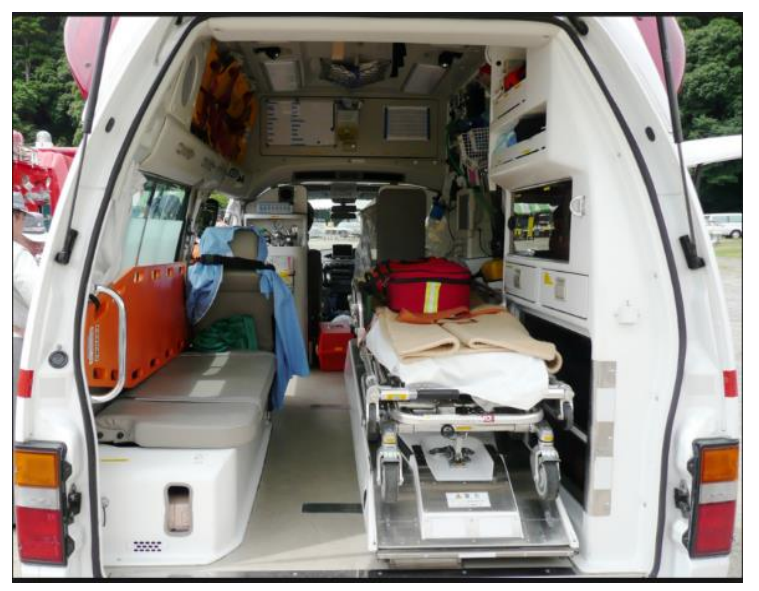

Fig.2. Ambulance Transport

In spite of these challenges, one good 'light in the tunnel' is the availability of network coverage for effective communication. With the proliferation of affordable mobile devices like mobile phones today, several people living in the rural areas are not exempted from owning them. Therefore, to bridge the communication gab when it comes to accessing needed healthcare in terms of availability of ambulances and the quick location of patients' position, it is cost-effective to take advantage of the benefits offered by ICT tools like the smartphones. This is consider important because, we believe that almost every household in rural areas has a phone and can access the Internet. Thus, having application system that run on mobile devices which can easily be used by patients could go a long way helping people living in rural areas like Mantsa village. As solution to the existing and unpleasant challenges faced by rural duelers in accessing needed medical facilities, this paper designed and implemented a mobile-based medical emergency ambulance scheduling system that is integrated with a LBS which is accessible via the Internet and wireless network on mobile device [3][5][15]. Thus, integrating mobile communication into the healthcare system can transform healthcare in the rural areas to a greater height.

\section{RELATED WORKS}

With the efforts to combat the challenges faced in the provision of ambulances to rural areas during emergency situations, this section highlights on some existing and similar systems in the perspective of mobile phones or devices. There are briefly outlined as follows:

Malusi and Kogeda [6] developed a mobile transportation system in 2012 to improve the hours of service needs and reduce the waiting time for transports. The system allows passengers to check if there is any transport coming as well as the availability of other passengers on the way and space. The system's performance was evaluated, proving $50 \%$ improved earnings due to operational efficiency. However, its primary focus was on improving public transportation and not the health services or ambulance transportation services. In a similar system, though patients-oriented, Global Rescue [8], an American-based company that provides medical advisory services, security and emergency flights worldwide employed the use of satellite communication to help remote patients in need of emergency services. It employed a satellite phone and one can subscribe. However, the person would have to inform the authority of their existing health problems so that it can be kept in their files as well as being given a first aid bag. In the event of emergency, by communicating via a satellite phone [9], the patient can be told on what to do using the equipment in the first aid bag, and evacuation to a hospital can be arranged if needed. Moreover, due to high cost of satellite phones and related transmission charges [7], some travelers to the remote regions relied on smart-phone applications and devices such as the De Lorme inReach which links a smart phone with a satellite via bluetooth. The limitation of the system is that it support only few text characters and if immediate medical assistance is needed, the texting doesn't work very efficiently. This could have been a better solution for the situation in the rural areas of Mafikeng, only if poverty was the challenge. Another similar system is GlobeFleet Ambulance Tracking and Task Management System [10]. It provides management solutions using mobile devices which allow for reporting, tracking and assignment.

In addition, the Ad-din Hospital in 2008 put in place a cost-effective ambulance service that utilized mobile phones and geographical positioning system (GPS) tracking. It was established to assist women in Dhaka, Bangladesh to have prompt access to emergency obstetric care [11]. It manages about 66 ambulances scattered throughout the city and an ambulance desk near Ad-din Hospital entrance. For efficiency and quality of service, each ambulance in the Ad-din network is equipped with a GPS tracking device to enable the dispatchers to determine which driver can reach a given patient with greatest ease and speed. However, the system does not offer direct communication between the patients or relatives and the paramedics. Also in the Northern Ireland, ambulance services are offered using computer-aided dispatch system which records all of the call-related details. This system is linked in with four other systems in which each perform interrelated but separate functions [12] such as gazetteer, the radio and telephony system, an automated vehicle location system and a mobile data 
terminal. The components operates at different control centers but cooperate to enable effective and efficient coordination of emergency call handling and response dispatch [13]. However, the challenge is the lack of integration between them. Moreover, people in need of emergency service still have to call and wait without knowing how far or how long it will take for the service providers to reach them.

Also, in the Western Cape Province of SA, when the paramedics receive a call requesting an ambulance, the caller is interrogated in order to determine the degree of the emergency. All emergency calls are prioritized and response is based on the nature of the incident which are classified as either priority 1 - immediate response (e.g. traffic injury, drowning, and acute MI), Priority 2 - As soon as possible (e.g. abdominal pain, fever, etc.) and Patient Transport (e.g. Clinic referral for consultation in outpatients) [8]. With this system, transport were provided for patients through a booking system and separate dispatchers, calls managed effectively, tracked and performance adjusted in real-time. In the North West province, Mafikeng, this system could probably be in existence but sometimes ambulance don't even show up. The reason could be that the degree of emergency are false due to how people responded to interrogations. Consequently, all responses and reported incidents are treated equally to reduce the phone conversation. Considering the nature of the current systems in the perspective of access to ambulance transportation by calls, the challenge is centered on the identification and treatment of emergency and non-emergency situations. The system itself is not accurate and the stated understanding of dispatch criteria to identify the nature and severity of patients' needs ranges from $68 \%$ to $99 \%$ in which a slight section of dispatch codes can reliably ascertain each situation [10]. Based on these challenges, it is clear that an improved system is needed that has the capacity to provide visual proof of the nature the emergency situations to service providers for appropriate actions to be taken to save patients' lives.

\section{Proposed SySTEM ARCHITECTURE}

In this section, we present an overview of the system and its architecture showing its components. They are discussed as follows:

\section{A. System Overview}

In this system, we used the technologies of mobile and cloud computing to provide a cost-effective means of communication between healthcare service providers and the patients in Mafikeng's rural areas. The system uses mobile (android) application coupled with GPS service to allow patients that need emergency services to request for ambulance and even book for hospital consultations. When used, the system has the capability to inform patients of predicted ambulance arrival time, reduce schedule preparation time, and improve communication between ambulance transport providers and the patients. This system is also intended to enhance ambulance transport operator and patient security by allowing quick location of the emergency scene and faster response. It also allow the patient to communicate with the healthcare providers or paramedics on their way to the rescue location. Moreover, the system has the benefits of reducing the long queues and waiting hours in hospitals which is based on appointments.

\section{B. System Architecture}

The architecture of the proposed system is shown in Fig. 3. The important components of the system are the computer system in the local unit of each hospital, the client software on smart mobile phones and 3G/4G wireless network, and a cloud-based server.

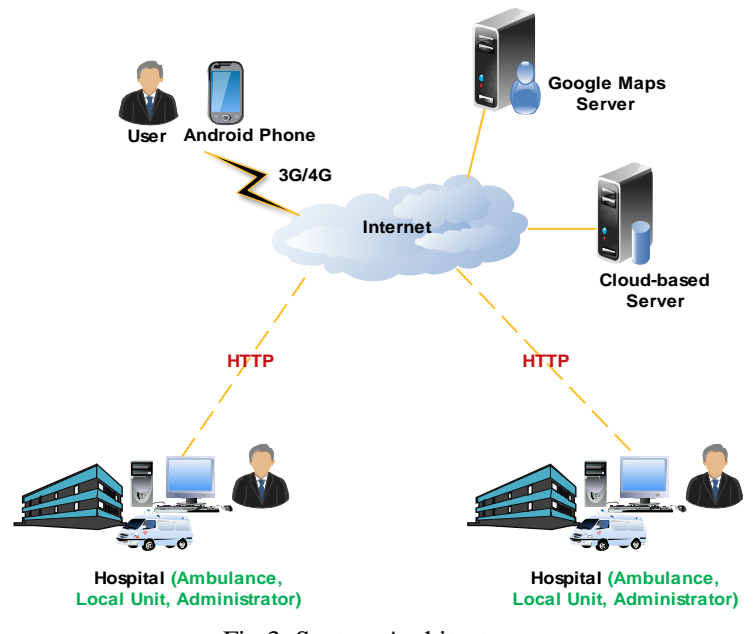

Fig.3. System Architecture

Computer system: This consist of the system components found at the local unit of the hospital. They include the computer which is connected to the cloudbased server via the Internet. Also, a web-based application software installed in the computer that provides interfaces with the functionalities that is needed to operate the system effectively. The software is integrated with GPS functionality using google maps to compute the distance between the patience and the ambulance or hospital and the optimal routes. Moreover, an operator called the administrator operates the system to effectively schedule available ambulances and paramedics during emergency situations.

Client software: This is the mobile application software that is installed on the client smart or android phones. With this application, patients are able to request for urgent ambulance service, book for hospital consultation and receive immediate feedback in terms of arrival time, delay time, and so on. This is done taking advantage of the mobile $3 \mathrm{G} / 4 \mathrm{G}$ wireless network connection which is available to all rural duelers in Mafikeng.

Cloud-based server: This is mainly a server that is located in the cloud where information is stored when patients ordered for ambulance or book for consultations using their phone. The computer in the local unit of the hospital always maintain active connection with the server to access patients request on real-time basis. Also, 
it allows ambulance drivers and paramedics to access patients request even if they are not in the hospital premises or if the local network in the hospital is down.

\section{ALGORITHM AND IMPLEMENTATION}

This section discusses the algorithmic design of the system, its operations and the implementation prototype.

\section{A. Algorithmic Design}

In order to ensure the optimal operation of the system to satisfy users' (patients, admin and paramedic) needs, we incorporated existing scheduling algorithm of First Come First Serve (FCFS) and the Dijkstra's algorithm [14]. The choice of the algorithms is to equip the system with the capabilities of handling several bookings and many emergency situations where several ambulance are needed but only few are available. With the FCFS algorithm, the queue of patients' requests are treated based on the principle of first to book first to be attended to. However, the distance to patient's location and the severity of patient's situation, cost and so on are not considered. Though is simple, it has implication on long varying and waiting time as well as increased costs.

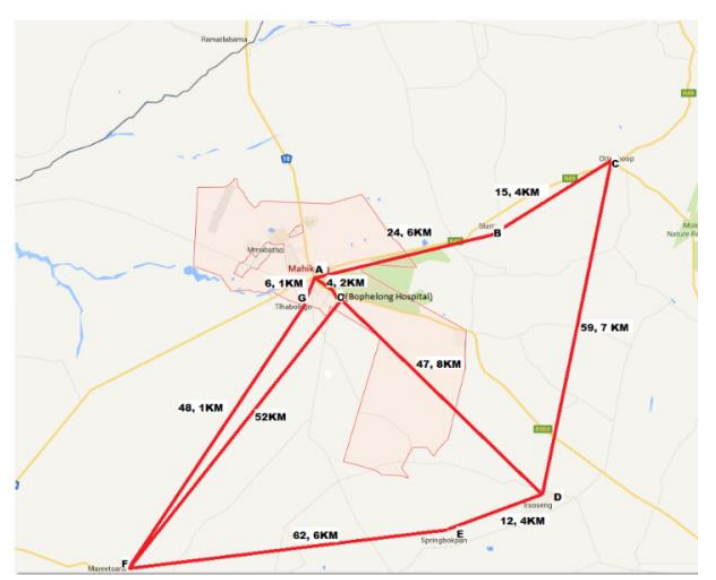

Fig.4. Rural areas around Bophelong Hospital in Mafikeng from Google Maps

On the other hand, the Dijkstra's algorithm offers the capability of computing the shortest paths [14], otherwise, the shortest route to patients' location considering the road networks. In this paper, the Dijkstra's algorithm is incorporated to assist paramedics to locate patients that need emergency service in the presence of limited resources. For instance, if only a single ambulance is available in a day and every patient's request is treated the same for transparency reasons, it is importance to handle such situations optimally. For instance, as shown in Fig. 4, considering that the only government hospital in Mafikeng represented by position A is the Bophelong Hospital situated in position $\mathrm{O}$ and patients from the rural villages of Slurry, Ottoshoop, Itsoseng, Springbokpan, Mareetsane and Tlhabologo in positions B, C, D, E, F, G respectively request for ambulance service at the same time when only one ambulance exist. In order to ensure that all patients are attended to at the possible shortest time, the Dijkstra's algorithm which is offered by the Google Maps is applied to compute the shortest route from one village to another irrespective of whose request is first. This in turn will save the time take to move from one village to another since Mafikeng has a scatted geographical locations and rural areas characterized by unstructured addresses.

\section{B. System Operations}

The system operates by having three important actors as users - the patients, the administrator and the paramedics. With the application installed on the patients' phone, he or she can interact with the system to either book for consultation or request for ambulance service. For the ambulance request, information such as name, the physical address and the nature of the emergency is given.

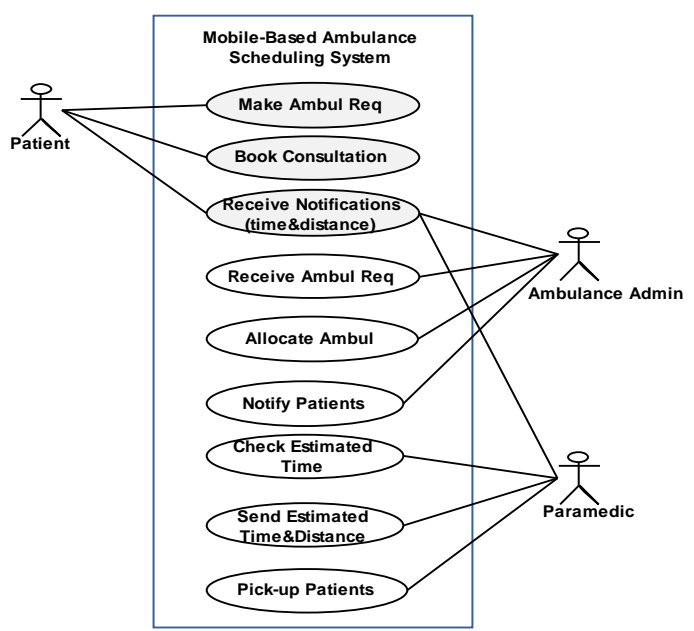

Fig.5. System use Cases

On the other hand, upon receipt of the request, the administrator uses the information stored in the cloud to allocate the optimal and available ambulance to attend to the patient as well as notify the available paramedic/driver immediately. On receipt of the notification, the paramedic then uses the available information via the Google Maps integrated with GPS service to estimate the best route to take, the distance between the current location and the patient's location as well as the estimated time taken to get there and pick up the patient. The possible interactions between the actors and the system is captured in the in the systems use cases shown in Fig. 5.

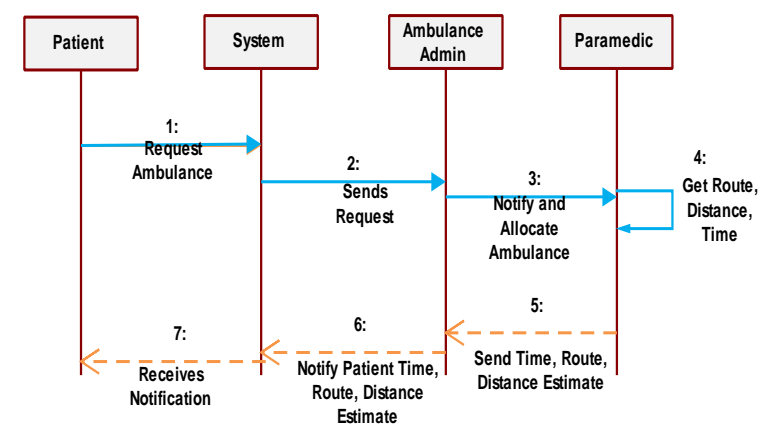

Fig.6. Ambulance Request Sequence 
Given the use cases in Fig. 5, taking the ambulance request into consideration, the simplified sequence of interactions between the patients and other system actors is captured in Fig. 6. The process of requesting for ambulance starts from (1) to (7). In this case, when the patient requests for an ambulance using his/her phone, the request is sent and stored in the cloud-based server. The ambulance administrator who operates just like the ambulance call center managers receives the request on a real-time basis and immediately checks the availability of an ambulance and allocates it accordingly. Immediately, a notification is sent to the paramedic available on duty to take charge of the emergency situation. In addition, the paramedic then uses the mobile interface to check the optimal route, distance and time and send notification to the ambulance administrator who then notifies the patient. The algorithmic flowchart for the process is shown in Fig. 7.

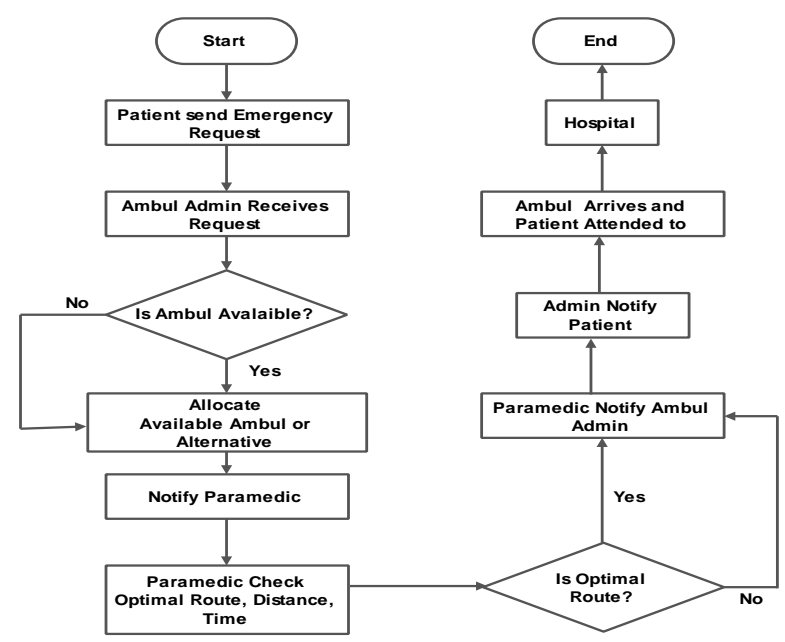

Fig.7. Algorithm of the System Operations

\section{System Prototype}

This section presents a system prototype that implements the process of emergency ambulance request in rural areas of Mafikeng of SA. The above stated idea is implemented using Google's Android Studio while incorporating Java JDK and XML. The choice of the technology is due to the flexibility it offers in both software design and development. Though, the process involve in the development is not easy using the technology, we believe the time invested in its usage will pay off in the long run in saving lives in the rural areas. The interfaces offered by the system are shown and discussed as follows:

The Patient's Interface: Based on the operation of the proposed system, this section presents the application interface that assist patients in their smooth request for ambulance during emergency situations. The client application home page is shown in Fig. 8.

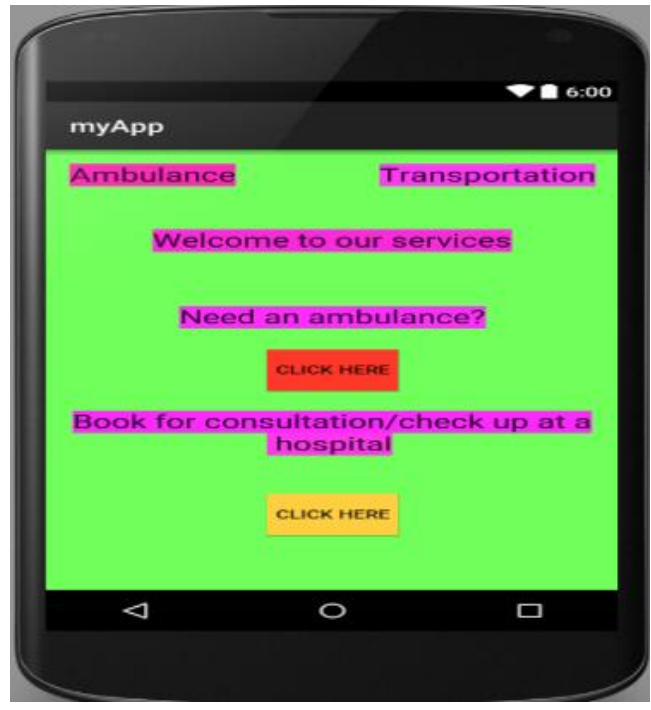

Fig.8. Client Application Home

As shown in Fig. 8, the client application home provides patients with the options of either requesting for an ambulance or booking for hospital consultation. For instance, if the patient's need is to request an ambulance for emergency, by clicking the appropriate button, the ambulance request page will appear as shown in Fig. 9.

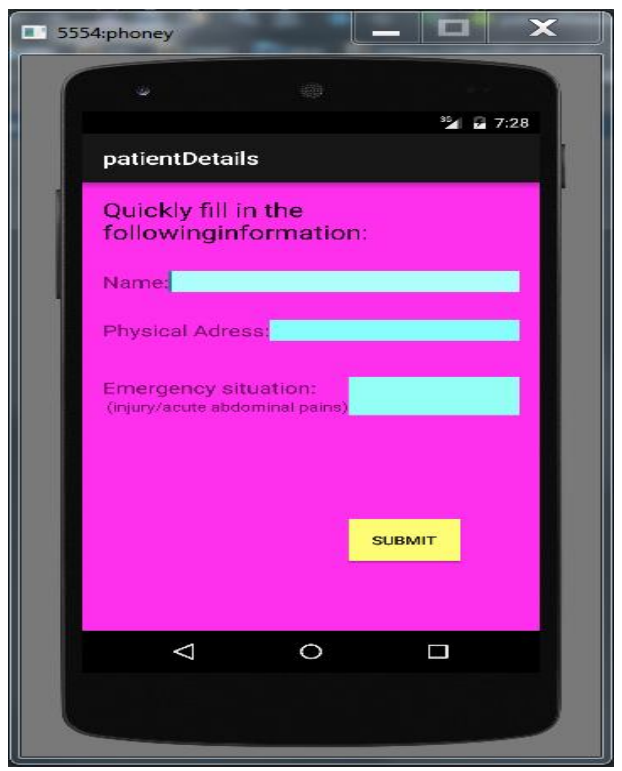

Fig.9. Ambulance Request

The interface shown in Fig. 9 is simple as patients' confidentiality will not be compromised even in the face of cloud security crisis. In this case, only the patient name, address and current situation is required to request an ambulance. However, if the need is to book for consultation, the same simple steps are required coupled with the patient's file number if already registered in the hospital, the nature of illness and time/date. See Fig. 10. 


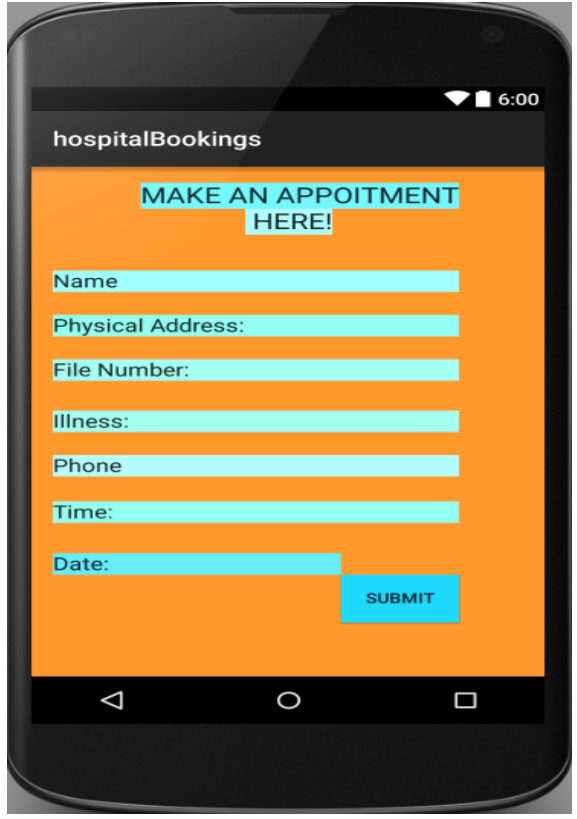

Fig.10. Booking for Consultation

In the case of ambulance request which is at the core of this paper, after a request is made, it is important that patients know the time it will take for the ambulance to arrive in order to prepare for urgent alternatives where necessary. Shown in Fig.11, the system will send the ambulance arrival time notification to the patient in that regard.

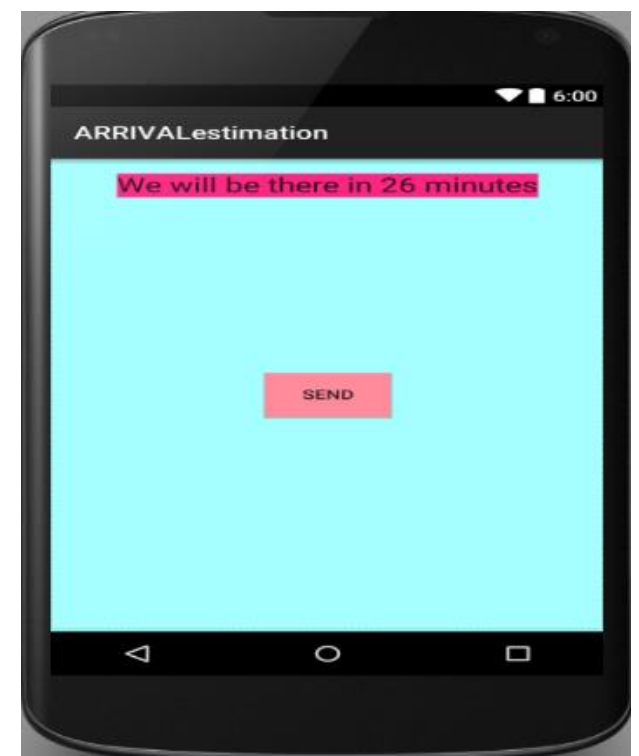

Fig.11. Estimation of Arrival Time Notification Received

Ambulance Admin and Paramedic Interfaces: Once ambulance requests are sent to the cloud-based server, it automatically notifies the ambulance administration which is accessible using mobile device or web browser on a desktop computer. Such notification will prompts the administrator to allocate available ambulance and notify the paramedics immediately. The interface of the notification received by the paramedic is captured in Fig. 12.

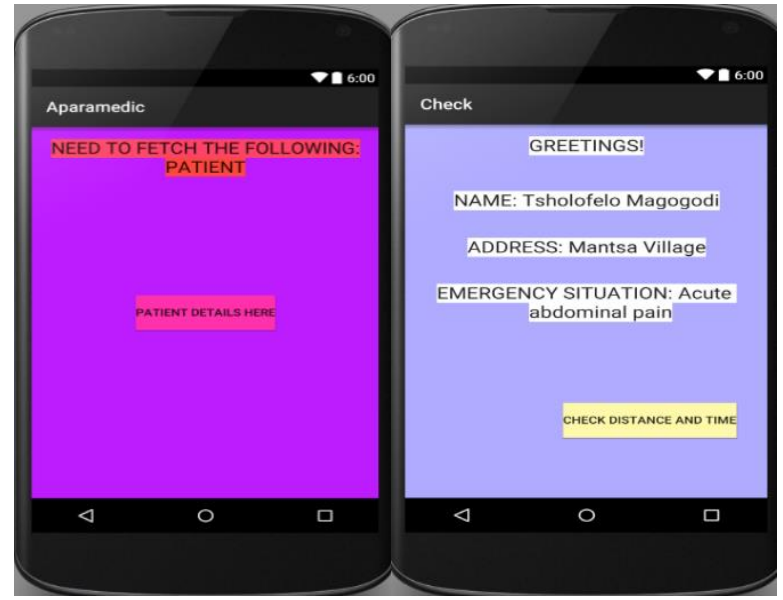

Fig.12. Notification and Patient'S Information Received by Paramedic

As shown on Fig. 12, once the "PATIENT DETAILS HERE" button is clicked, the information shown on the right screen will be displayed. Consequently, the paramedic will click the button "CHECK DISTANCE AND TIME", which is linked to Google Maps. With this functionality, the paramedic can compute the optimal route for the request. That is, can check for the estimated distance and time between the current position and the patient's location. This is presented in Fig. 13 which shows a request between a patient in Mantsa village and Bophelong Hospital in Mafiking where the possible optimal route is $24.1 \mathrm{Km}$ and $34 \mathrm{~min}$. The obtained information is then sent to the ambulance administrator which is forwarded to patients as shown in Fig. 11.

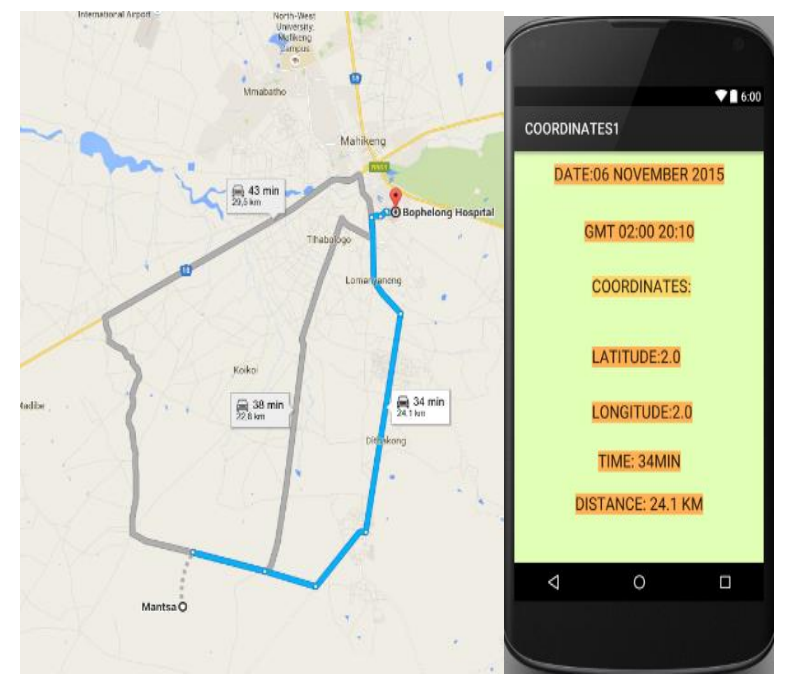

Fig.13. Google Maps Extract of Distance and Time between Bophelong Hospital and Mantsa Village

\section{DISCUSSIONS}

Today, the rural areas in Mafikeng are faced with many challenges or adversities where lack of basic public facilities like Emergency Medical Services (EMSs) and so on exacerbated by poverty is not an exception. A particular case is the people of Mantas village where the access to the hospitals or health centers irrespective of the 
life-threatening nature of the problems that requires emergencies is still at large. Therefore, such situations have to be rescued. We observed that the factors that affects having access to healthcare system especially in the face of emergencies are communication and transportation. It is difficult to call emergency centers and roads are bad or addresses unstructured leading to nonarrival of ambulances on time and even deaths may occurred. Given the critical context, the system we proposed in this paper is capable of providing solutions to challenges being faced by those villagers. The system takes advantage of android mobile applications technologies, the availability of mobile phones in every household coupled with cloud storage technology to offer cost-effective services for patients in the rural areas.

The main benefits of this system are providing improved-communication between patients and health providers such as the ambulance driver, paramedics, doctors, nurses and so on. Moreover, it reduces the time spent waiting for the ambulance and long queues in hospital by providing location-based ambulance transportation and hospital coordination system that allows coordination and tracking of ambulance, hospital and the actual location of the patient. Moreover, the system allows patients to do timely booking of consultations appointment without having to go and stand in long queues at the hospital. It is simple, user-friendly and no registration is required. In addition, patients will no longer have the problems of calling emergency centers but simply send online messages which is received on real time mode. High computing skills are not required for the system but at least, the person should be able to operate the phone, write his or her name and address.

\section{CONCLUSIONS}

This paper identified and presented the challenges people in the rural areas of Mafikeng faced with respect to public health care facilities like medical emergency services. In particular, patients found it difficult if not impossible to access medical ambulance transport during emergency situations which consequently, leads to loss of lives that could be prevented. With such challenges and others, this paper proposed a solution in the capacity of Mobile-based ambulance transportation scheduling system. It is aim at improving ambulance transportation availability and other services when requests are made without having to call or wait for long time. The paper analyzed, designed and implemented a prototype to demonstrate the operation of the system to show its effectiveness with respect to the current systems. Based on its mode of operations, we believe that if adopted for used by people living in the rural areas, not only Mafikeng rural areas but areas where modern hospital or health facilities are not located in SA. Moreover, it could go a long way to make their lives easier and prevents the loss of lives of thousands of people needing emergencies. Our future work will be to improve the system by incorporating technologies of Internet of things (IoT) to scale it up and link it with all nearby hospitals such that if a service is not available in one, another hospital that offer such service can be suggested immediately alongside its distance, arrival time and the availability of paramedics, doctors and so on to attend to the emergency situation.

\section{ACKNOWLEDGMENT}

Our sincere appreciation goes to staff members and the honors students of the Department of Computer Science, North-West University, Mafikeng campus for the successful completion of this research.

\section{REFERENCES}

[1] Barbeau, S.J., Labrador, M.A., Winters, P.L., Pérez, R., and Georggi, N.L.: 'Location API 2.0 for J2ME-A new standard in location for Java-enabled mobile phones', Computer Communications, 31, (6), pp. 1091-1103, 2008.

[2] Cooke, R.: 'The role and impact of transport on rural communities accessing the state health care system in south africa', rural health advocacy project, 2013

[3] Phillips, A., Schroth, F., Palmer, G.M., Zielinski, S.G., Smith, A.P., and Cunningham. 'Location-based services', Google Patents, 2010.

[4] Siruma, A., Hornby, D., Srinivas, S. An Assessment of Maternal Health Issues in Two Villages in the Eastern Cape Province of South Africa. Int. J. Environ. Res. Public Health 2014, 11, 9871-9884

[5] Junglas, I.A., and Watson, R.T.: 'Location-based services', Communications of the ACM, 2008, 51, (3), pp. 65-69

[6] Malusi, Y., and Kogeda, O.: 'A mobile transport scheduling and coordination system for marginalized rural areas'. pp. 10-13, 2013

[7] DeLone, W.H., and McLean, E.R.: 'Information systems success: The quest for the dependent variable', Information systems research, 1992, 3, (1), pp. 60-95

[8] Hosokawa, M.: 'Disaster risk evaluation and damage detection using remote sensing data for global rescue operations', 2008

[9] Hosokawa, M., Jeong, B.-p., and Takizawa, O.: 'Earthquake intensity estimation and damage detection using remote sensing data for global rescue operations', IEEE, 2009.

[10] Globefleet: 'GManager Ambulance' http://www.europages.com/filestore/gallery/15/e1/782954 5_c3deb03d.pdf. accessed: 18 August, 2015.

[11] Ngongo, C.: 'A low-cost ambulance network to improve access to maternity services in Dhaka Bangladesh' 2012 EngenderHealth,

https://www.engenderhealth.org/files/pubs/fistula-caredigital-archive/2/2.1/bangladesh_ambulance_brief.pdf, accessed: 14 August, 2015

[12] Heo, Y.H., Choi, S.H., Lee, J.H., Kwak, Y.J., and Jeong, K.I.: 'Method of transmitting scheduling information on an enhanced uplink dedicated channel in a mobile communication system', 2006

[13] Marinovich, A., Afilalo, J., Afilalo, M., Colacone, A., Unger, B., Giguere, C., Leger, R., Xue, X., and MacNamara, E.: 'Impact of ambulance transportation on resource use in the emergency department', Academic emergency medicine, 2004, 11, (3), pp. 312-315

[14] Yan,M.: 'Dijkstra's Algorithm", available on http://www.cs.princeton.edu.algsDS07, accessed: 11 July 2015.

[15] Virrantaus, K., Markkula, J., Garmash, A., Terziyan, V., 
Alainen, J.V., Katanosov, A., and Tirri, H.: 'Developing GIS-supported location-based services', IEEE, 2001, pp. 66-75.

\section{Authors' Profiles}

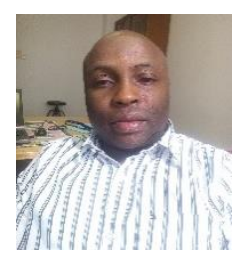

Isong Bassey received B.Sc. degree in Computer Science from the University of Calabar, Nigeria in 2004 and M.Sc. degrees in Computer Science and Software Engineering from Blekinge Institute of Technology, Sweden in 2008 and 2010 respectively. Moreover, he received a $\mathrm{PhD}$ in Computer Science in the North-West University, Mafikeng Campus, South Africa in 2014. Between 2010 and 2014 he was a faculty member of the University of Venda, South Africa and a Lecturer of Computer Science and Information Systems. Currently, he is a Lecturer in the Department of Computer Sciences, Mafikeng Campus, North-West University. He is also a member of IEEE Computer and Communication Societies. His research interests include Software Engineering,
Requirements Engineering, Software Maintenance, Cybersecurity, Software-Defined Networks, Cloud and Mobile Computing, ICT4D and Computer Science Education.

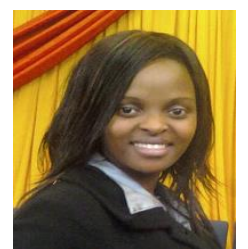

Nosipho Dladlu obtained her B.Sc. (Hons) and M.Sc. degrees in Computer Science from the North-West University, Mafikeng, South Africa in 2011 and 2014 respectively. Currently, she is a Lecturer in the Department of Computer Sciences and a Faculty member of FAST, North-West University, Mafikeng Campus. Her research interests include: Cloud Computing, Mobile Computing, Networks and HCI.

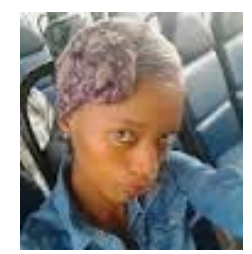

Tsholofelo Magogodi is a postgraduate student of the department of Department of Computer Sciences, FAST, North-West University, Mafikeng Campus. Her research interests include: Cloud Computing, Mobile Computing and eHealth.

How to cite this paper: Bassey Isong, Nosipho Dladlu, Tsholofelo Magogodi,"Mobile-Based Medical Emergency Ambulance Scheduling System", International Journal of Computer Network and Information Security(IJCNIS), Vol.8, No.11, pp.14-22, 2016.DOI: 10.5815/ijcnis.2016.11.02 